\title{
STRONG KLEE-ANDÔ THEOREMS THROUGH AN OPEN MAPPING THEOREM FOR CONE-VALUED MULTI-FUNCTIONS
}

\author{
MIEK MESSERSCHMIDT
}

\begin{abstract}
A version of the classical Klee-Andô Theorem states the following: For every Banach space $X$, ordered by a closed generating cone $C \subseteq X$, there exists some $\alpha>0$ so that, for every $x \in X$, there exist $x^{ \pm} \in C$ so that $x=x^{+}-x^{-}$and $\left\|x^{+}\right\|+\left\|x^{-}\right\| \leq \alpha\|x\|$.

The conclusion of the Klee-Andô Theorem is what is known as a conormality property.

We prove stronger and somewhat more general versions of the Klee-Andô Theorem for both conormality and coadditivity (a property that is intimately related to conormality). A corollary to our result shows that the functions $x \mapsto x^{ \pm}$, as above, may be chosen to be bounded, continuous, and positively homogeneous, with a similar conclusion yielded for coadditivity. Furthermore, we show that the Klee-Andô Theorem generalizes beyond ordered Banach spaces to Banach spaces endowed with arbitrary collections of cones. Proofs of our Klee-Andô Theorems are achieved through an Open Mapping Theorem for cone-valued multi-functions/correspondences.

We very briefly discuss a potential further strengthening of The Klee-Andô Theorem beyond what is proven in this paper, and motivate a conjecture that there exists a Banach space $X$, ordered by a closed generating cone $C \subseteq X$, for which there exist no Lipschitz functions $(\cdot)^{ \pm}: X \rightarrow C$ satisfying $x=x^{+}-x^{-}$ for all $x \in X$.
\end{abstract}

\section{INTRODUCTION}

Let $X$ be a Banach space and $\left\{C_{\omega}\right\}_{\omega \in \Omega}$ a collection of closed cones in $X$, indexed by a set $\Omega$. Consider the following two geometric propertie $\mathbb{1}\left\{C_{\omega}\right\}_{\omega \in \Omega}$ could satisfy in $X$ :

(1) Conormality: There exists a constant $\alpha>0$ such that, for every $x \in X$, there exists a decomposition $x=\sum_{\omega \in \Omega} c_{\omega}$ with $\sum_{\omega \in \Omega}\left\|c_{\omega}\right\| \leq \alpha\|x\|$ and $c_{\omega} \in C_{\omega}$ for all $\omega \in \Omega$.

(2) Coadditivity: For some normed subspace $Z$ of $X^{\Omega}$, there exists a constant $\alpha>0$ such that, for every $\xi \in Z$, there exists some $x \in \bigcap_{\omega \in \Omega}\left(\xi_{\omega}+C_{\omega}\right)$ with $\|x\| \leq \alpha\|\xi\|$.

2010 Mathematics Subject Classification. 46B20, 46A30 (primary), and 46B40, 32A12 (secondary).

The author's research was funded by The Claude Leon Foundation.

${ }^{1}$ Historically the properties "conormality" and "coadditivity" are respectively called " $\alpha$ generating" and " $\alpha$-directedness". Our reason for deviating is in favor of the mnemonic device connecting "conormality" and "coadditivity" to their dual properties "normality" and "additivity" (standard terms which we do not define or need in this paper): Roughly, a space is normal (additive) if and only if its dual is conormal (coadditive), and vice versa. The interested reader is referred to [10] and 11] which tries to reference the previously existing literature on such dualities as completely as possible. 
The following two very simple examples are easily seen to be conormal and coadditive respectively, and also illustrates that these properties make sense even outside the realm of classical ordered Banach spaces.

Example 1.1. For $\mathbb{R}^{2}$ with the Euclidean norm with $\left\{e_{1}, e_{2}\right\}$ the standard basis for $\mathbb{R}^{2}$, set $\Omega:=\left\{e_{1}, e_{2},-\left(e_{1}+e_{2}\right)\right\} \subseteq \mathbb{R}^{2}$, and define the cones $\left\{C_{x}\right\}_{x \in \Omega}$ by setting $C_{x}:=\{\lambda x \mid \lambda \geq 0\}$ for all $x \in \Omega$. The space $\mathbb{R}^{2}$ with $\left\{C_{x}\right\}_{x \in \Omega}$ is easily seen to be conormal.

Example 1.2. For $\mathbb{R}^{2}$ with the Euclidean norm, we will define the cones $C_{1}:=$ $\left\{(x, y) \in \mathbb{R}^{2} \mid x, y \geq 0\right\}$ and $C_{2}:=\left\{\alpha(1,1)+\beta(1,-1) \in \mathbb{R}^{2} \mid \alpha, \beta \geq 0\right\}$. The space $\mathbb{R}^{2}$ with $\left\{C_{j}\right\}_{j \in\{1,2\}}$ is easily seen to be coadditive (with $Z$ taken as the $\ell^{2}$-direct sum two copies of $\mathbb{R}^{2}$ ).

The following result dates back to Andô's [4, Lemma 1], which was proven by employing an Open Mapping Theorem due to Klee [8, (3.2)]. The reader should recognize the conclusion of the following result as a conormality property.

Theorem 1.3 (Klee-Andô Theorem [4, Lemma 1], 8, (3.2)]). Let $X$ be a Banach space ordered by a closed cone $C \subseteq X$. If $C$ is generating, i.e., $X=C-C$, then there exists a constant $\alpha>0$ so that, for every $x \in X$, there exists a decomposition $x=x^{+}-x^{-}$with $x^{ \pm} \geq 0$ and $\left\|x^{+}\right\|+\left\|x^{-}\right\| \leq \alpha\|x\|$.

Recently, a stronger version of the Klee-Andô Theorem, stated as Corollary 5.6 below, was proven by the author and de Jeu in [7. Its proof employed a generalization of Banach's classical Open Mapping Theorem [7, Theorem 3.2] together with Michael's Selection Theorem which stated in this paper as Theorem5.3. We note that Corollary 5.6 has wider applicability than ordered Banach spaces (cf. Example 1.1) and has a distinctly more geometrical flavor than the order theoretic Theorem 1.3 (where we essentially restrict our attention to only two cones: $C$ and $-C)$.

Corollary 5.6 (Strong Klee-Andô Theorem for conormality [7, Theorem 4.1]). Let $X$ be a Banach space and $\left\{C_{\omega}\right\}_{\omega \in \Omega}$ an indexed collection of closed cones in $X$. The following are equivalent:

(1) For every $x \in X$, there exists a decomposition $x=\sum_{\omega \in \Omega} c_{\omega}$, with $c_{\omega} \in C_{\omega}$ for every $\omega \in \Omega$, and satisfying $\sum_{\omega \in \Omega}\left\|c_{\omega}\right\|<\infty$.

(2) There exists an $\alpha>0$ such that, for every $x \in X$, there exists a decomposition $x=\sum_{\omega \in \Omega} c_{\omega}$, with $c_{\omega} \in C_{\omega}$ for every $\omega \in \Omega$, and satisfying $\sum_{\omega \in \Omega}\left\|c_{\omega}\right\| \leq \alpha\|x\|$.

(3) There exists an $\alpha>0$ and, for every $\omega \in \Omega$, there exists a continuous positively homogeneous map $\delta_{\omega}: X \rightarrow C_{\omega}$ such that, for every $x \in X$, we have $x=\sum_{\omega \in \Omega} \delta_{\omega}(x)$ and $\sum_{\omega \in \Omega}\left\|\delta_{\omega}(x)\right\| \leq \alpha\|x\|$.

In other words, the mere fact that one can decompose arbitrary elements of $X$ as the limit of absolutely convergent series with terms chosen from the closed cones $\left\{C_{\omega}\right\}_{\omega \in \Omega}$, automatically implies that one can always choose such a decomposition in a bounded, continuous and positively homogeneous and manner. This is, of course, particularly useful when considering spaces of continuous functions taking values in an ordered Banach space (cf. [6, Corollary 2.8]). 
Having now discussed the Strong Klee-Andô Theorem for conormality, we point out that coadditivity and conormality are intimately related, in that both their statements satisfy the following general template:

For every structure $A$ of a certain type, there exists some related structure $B$, and this $B$ is bounded, in some sense, by $A$.

This relationship and the fact that the Klee-Andô Theorem for conormality was previously established in [7, raises the following question:

Question 1.4. Does there there exist a Klee-Andô type theorem for coadditivity? Roughly, if the intersection of certain translates of a collection of closed cones in a Banach space is always non-empty, can one always find an element in such an intersection in a bounded, continuous and positively homogeneous manner?

We will answer this question positively in this paper by proving Corollary 5.5.

Corollary $\mathbf{5 . 5}$ (Strong Klee-Andô Theorem for coadditivity). Let $X$ be a Banach space and $\left\{C_{\omega}\right\}_{\omega \in \Omega}$ an indexed collection of closed cones in $X$. Let $Z$ be either of the spaces $\mathbf{c}(\Omega, X)$ or $\ell^{\infty}(\Omega, X)$. Then the following are equivalent:

(1) For every $\xi \in Z$, the intersection $\bigcap_{\omega \in \Omega}\left(\xi_{\omega}+C_{\omega}\right)$ is non-empty.

(2) There exists an $\alpha>0$ such that, for every $\xi \in Z$, there exists some $y \in \bigcap_{\omega \in \Omega}\left(\xi_{\omega}+C_{\omega}\right)$ with $\|y\| \leq \alpha\|\xi\|_{\infty}$.

(3) There exists an $\alpha>0$ and a continuous positively homogeneous map $v$ : $Z \rightarrow X$ such that, for every $\xi \in Z$, we have $v(\xi) \in \bigcap_{\omega \in \Omega}\left(\xi_{\omega}+C_{\omega}\right)$ and $\|v(\xi)\| \leq \alpha\|\xi\|_{\infty}$.

Our approach in proving Corollary [5.5 is similar in broad strokes to the proof of [7. Theorem 4.1], stated in this paper as Corollary5.6. However, the Open Mapping Theorem [7, Theorem 3.2] employed in the proof of [7, Theorem 4.1] is not strong enough to establish the results leading up to Corollary 5.5. We further strengthen [7. Theorem 3.2] to the version stated as Theorem 4.5, which we use to establish both our Strong Klee-Andô Theorems for both coadditivity and conormality:

Theorem 4.5 (Open Mapping Theorem for cone-valued correspondences). Let $C$ a complete metric cone (as defined in Section 4), Y a Banach space and $D \subseteq Y$ a closed cone. Let $T: C \rightarrow Y$ be a continuous additive positively homogeneous map. If the correspondence $\Psi: C \rightarrow Y$ defined by $\Psi(c):=T c+D(x \in C)$ is surjective (in the sense that, for every $y \in Y$, there exists some $c \in C$ such that $y \in \Psi(c)$ ), then the image under $\Psi$ of the open unit ball about zero in $C$ is open in $Y$.

We point out the following problem toward further strengthening of the KleeAndô Theorems proven in this paper. This problem is motivated by the observation that the lattice operations in every Banach lattice are Lipschitz [13, Proposition II.5.2], and the further question as to whether a form of this carries over to ordered Banach spaces, or more generally, to Banach spaces endowed with arbitrary collections of cones. To the author's knowledge, this problem remains open:

Problem 1.5. Do Corollaries 5.5 and 5.6 remain true when, in their statements, the word "continuous" is replaced by the word "Lipschitz"?

A straightforward application of [12, Corollary 4.6] shows that Corollaries 5.5] and 5.6 remain true when, in their statements, the word "continuous" is replaced 
with the phrase "continuous and pointwise Lipschitz on a dense set of its domain". However, an example devised by Aharoni and Lindenstrauss (cf. 1] and [5, Example 1.20]) shows that [12, Corollary 4.6] cannot be improved, in general, to a result yielding functions that are Lipschitz. We refer the reader to [12] for more detail.

The above-mentioned example by Aharoni and Lindenstrauss and the difficulties presented in attempts at solving Problem 1.5 positively, motivates the following conjecture which, if true, is sufficient to solve Problem 1.5 negatively:

Conjecture 1.6. There exists a Banach space $X$, ordered by a closed generating cone $C \subseteq X$, for which there exist no Lipschitz functions $(\cdot)^{ \pm}: X \rightarrow C$ satisfying $x=x^{+}-x^{-}$for all $x \in X$.

We briefly describe the structure of the paper.

In Section 2 we provide some preliminary definitions and notation used throughout the current paper.

Sections 3 and 4 sees the introduction of some terminology on correspondences (also known as multi-functions) and will prove some general results. Section 3 will introduce what we call "additive" and " $\alpha$-bounded" correspondences and will establish some general results that we will use in later sections. Section 4 sees the definition of metric cones and proof of one of our main results, an Open Mapping Theorem for cone-valued correspondences (Theorem 4.5).

We apply our results from the previous sections to prove our Strong Klee-Andô Theorems for conormality and coadditivity in Section 5 Although our Strong KleeAndô Theorem for conormality was previously established in [7, we also include a proof of it here for the sake of completeness.

\section{Preliminary Definitions and notation}

All vector spaces are assumed to be over the reals.

Let $V$ be a vector space. A non-empty subset $C \subseteq V$ satisfying $C+C \subseteq C$ and $\lambda C \subseteq C$ for all $\lambda \geq 0$ will be called a cone. If $C \cap-C=\{0\}$, then we will say $C$ is a proper cone. If $V=C-C$, then we will say that $C$ is generating in $V$. Translation invariant and positively homogeneous pre-orders (partial orders) on $V$ are easily seen to be in bijection with cones (proper cones) in $V$, cf. [3, Section 1.1]. We will say $V$ is ordered by a cone $C$ by defining " $v \leq w$ " to mean $w \in v+C$ for $v, w \in V$.

Let $\phi: V \rightarrow \mathbb{R}$ be any map. The map $\phi$ is said to be positively homogeneous, if $\phi(\lambda v)=\lambda \phi(v)$ for all $v \in V$ and $\lambda \geq 0$. The map $\phi$ is said to be subadditive if $\phi(v+w) \leq \phi(v)+\phi(w)$ for all $v, w \in V$.

For a normed space $X$, we will denote the unit sphere, closed unit ball and open unit ball of $X$ respectively by $\mathbf{S}_{X}, \mathbf{B}_{X}$ and $\mathbb{B}_{X}$.

Let $\Omega$ be an arbitrary index set, and let $X$ be a Banach space.

(1) For $1 \leq p \leq \infty$, by $\ell^{p}(\Omega, X)$ we will denote the usual $\ell^{p}$-direct sum of $|\Omega|$ copies of $X$, with the norm on $\ell^{p}(\Omega, X)$ denoted by $\|\cdot\|_{p}$.

(2) By $\mathbf{c}(\Omega, X)$ we will denote the closed subspace of $\ell^{\infty}(\Omega, X)$ of all elements $\xi \in \ell^{\infty}(\Omega, X)$ for which there exists some $x \in X$ such that, for every $\varepsilon>0$, the set $\left\{\omega \in \Omega \mid\left\|\xi_{\omega}-x\right\| \geq \varepsilon\right\}$ is finite.

Let $\left\{C_{\omega}\right\}_{\omega \in \Omega}$ be an indexed collection of cones in $X$, and let $Z$ be some vector subspace of $X^{\Omega}$. The notation " $\bigoplus_{\omega \in \Omega} C_{\omega} \subseteq Z$ " will be used to denote the set $\left\{\xi \in Z \mid \forall \omega \in \Omega, \xi_{\omega} \in C_{\omega}\right\}$. 


\section{BOUNDED AND ADDITIVE CORRESPONDENCES}

In this section our goal is to prove the general result, Proposition 3.3, which establishes the lower hemicontinuity of certain correspondences constructed from given correspondences having some extra algebraic structure.

We first introduce some terminology: Let $A, B$ be sets. By a correspondence we mean a set valued map $\varphi: A \rightarrow 2^{B}$ and will use the notation $\varphi: A \rightarrow B$. If $A$ and $B$ are topological spaces, we will say that a correspondence $\varphi: A \rightarrow B$ is lower hemicontinuous if, for every $a \in A$ and every open set $U \subseteq B$ satisfying $\varphi(a) \cap U \neq \emptyset$, there exists some open set $V \ni a$ satisfying $\varphi(v) \cap U \neq \emptyset$ for all $v \in V$. By a continuous selection of $\varphi$ we mean a continuous function $f: A \rightarrow B$ with $f(a) \in \varphi(a)$ for all $a \in A$.

Definition 3.1. Let $X$ and $Y$ be normed spaces and let $\varphi: X \rightarrow Y$ be a correspondence.

(1) We will say $\varphi$ is additive if, for $x, z \in X, \varphi(x)+\varphi(z) \subseteq \varphi(x+z)$.

(2) For some $\alpha>0$, we will say $\varphi$ is $\alpha$-bounded if, for every $x \in X$ and $\varepsilon>0$, $\varphi(x) \cap(\alpha+\varepsilon)\|x\| \mathbf{B}_{Y}$ is non-empty.

We begin with the following lemma, which is a crucial ingredient in the proof of Proposition 3.3

Lemma 3.2. Let $X$ be a normed space. Let $\alpha>0$ and $G \subseteq X$ be a convex set such that, for every $\varepsilon>0$, the set $G \cap(\alpha+\varepsilon) \mathbf{B}_{X}$ is non-empty. If, for an open set $U \subseteq X$ and some $\varepsilon_{0}>0$, the set $G \cap\left(\alpha+\varepsilon_{0}\right) \mathbf{B}_{X} \cap U$ is non-empty, then $G \cap\left(\alpha+\varepsilon_{0}\right) \mathbb{B}_{X} \cap U$ is also non-empty.

Proof. Let $U \subseteq X$ be open and $\varepsilon_{0}>0$ such that $G \cap\left(\alpha+\varepsilon_{0}\right) \mathbf{B}_{X} \cap U \neq \emptyset$. Let $x \in G \cap\left(\alpha+\varepsilon_{0}\right) \mathbf{B}_{X} \cap U$. If $x \in\left(\alpha+\varepsilon_{0}\right) \mathbb{B}_{X}$, then we are done. We therefore assume that $x \in\left(\alpha+\varepsilon_{0}\right) \mathbf{S}_{X}$. Let $y \in G \cap\left(\alpha+2^{-1} \varepsilon_{0}\right) \mathbf{B}_{X} \neq \emptyset$. Then, for every $t \in(0,1]$,

$$
\begin{aligned}
\|t y+(1-t) x\| & \leq t\|y\|+(1-t)\|x\| \\
& \leq t\left(\alpha+2^{-1} \varepsilon_{0}\right)+(1-t)\left(\alpha+\varepsilon_{0}\right) \\
& <t\left(\alpha+\varepsilon_{0}\right)+(1-t)\left(\alpha+\varepsilon_{0}\right) \\
& =\left(\alpha+\varepsilon_{0}\right) .
\end{aligned}
$$

In other words, $t y+(1-t) x \in\left(\alpha+\varepsilon_{0}\right) \mathbb{B}_{X}$ for all $t \in(0,1]$. Since $[0,1] \ni t \mapsto t y+(1-$ $t) x$ is continuous, there exists some $t_{0} \in(0,1]$ such that $t_{0} y+\left(1-t_{0}\right) x \in U$. Since $G$ is convex, $t_{0} y+\left(1-t_{0}\right) x \in G$. We conclude $t_{0} y+\left(1-t_{0}\right) x \in G \cap\left(\alpha+\varepsilon_{0}\right) \mathbb{B}_{X} \cap U$.

Proposition 3.3. Let $X$ and $Y$ be normed spaces and $\alpha>0$. Let $\varphi: X \rightarrow Y$ be a convex-valued additive $\alpha$-bounded correspondence. Then, for every $\varepsilon>0$, the correspondence $\varphi_{\varepsilon}: \mathbf{S}_{X} \rightarrow Y$, defined by

$$
\varphi_{\varepsilon}(x):=\varphi(x) \cap(\alpha+\varepsilon) \mathbf{B}_{Y} \quad\left(x \in \mathbf{S}_{X}\right),
$$

is non-empty-and convex-valued and lower hemicontinuous.

Proof. Since $\varphi$ is $\alpha$-bounded and convex-valued, that $\varphi_{\varepsilon}$ is non-empty- and convexvalued for every $\varepsilon>0$ is immediate.

We establish the lower hemicontinuity of $\varphi_{\varepsilon}$. Let $\varepsilon>0$ and $x \in \mathbf{S}_{X}$ be arbitrary. Let $U \subseteq Y$ be open such that $\varphi_{\varepsilon}(x) \cap U=\varphi(x) \cap(\alpha+\varepsilon) \mathbf{B}_{Y} \cap U$ is non-empty. By Lemma 3.2. $\varphi(x) \cap(\alpha+\varepsilon) \mathbb{B}_{Y} \cap U$ is non-empty. Let $y \in \varphi(x) \cap(\alpha+\varepsilon) \mathbb{B}_{Y} \cap U$ be 
arbitrary, and let $r>0$ be such that $y+r \mathbb{B}_{Y} \subseteq(\alpha+\varepsilon) \mathbb{B}_{Y} \cap U$. Now, let $z \in \mathbf{S}_{X}$ be such that $\|z-x\|<r(\alpha+\varepsilon)^{-1}$. Since $\varphi$ is $\alpha$-bounded, there exists some $v \in$ $\varphi(z-x) \cap(\alpha+\varepsilon)\|z-x\| \mathbf{B}_{Y}$. Then, $\|v\| \leq(\alpha+\varepsilon)\|z-x\|<r(\alpha+\varepsilon)^{-1}(\alpha+\varepsilon)=r$, so that $y+v \in y+r \mathbb{B}_{Y} \subseteq(\alpha+\varepsilon) \mathbb{B}_{Y} \cap U$, and, since $\varphi$ is additive, we have $y+v \in \varphi(x+z-x)=\varphi(z)$. Therefore $y+v \in \varphi(z) \cap(\alpha+\varepsilon) \mathbb{B}_{Y} \cap U=\varphi_{\varepsilon}(z) \cap U$. Since $z$ was chosen arbitrarily from $V:=\left(x+r(\alpha+\varepsilon)^{-1} \mathbb{B}_{X}\right) \cap \mathbf{S}_{X}$, we conclude that $\varphi_{\varepsilon}$ is lower hemicontinuous.

\section{An Open Mapping Theorem for COne-Valued CORRespondences}

In this section we will prove one of our main results, namely an Open Mapping Theorem for cone-valued correspondences.

We begin with the following definitions and notation:

Definition 4.1. Let $C$ be a set equipped with operations $+: C \times C \rightarrow C$ and - : $\mathbb{R}_{\geq 0} \times C \rightarrow C$. The set $C$ will be called an abstract cone, if there exists an element $0 \in C$ such that, for all $u, v, w \in C$ and $\lambda, \mu \in \mathbb{R}_{\geq 0}$, the following hold:

(1) $u+0=u$

(2) $(u+v)+w=u+(v+w)$

(3) $u+v=v+u$

(4) $u+v=u+w$ implies $v=w$

(5) $1 u=u$

(6) $(\lambda \mu) u=\lambda(\mu u)$

(7) $(\lambda+\mu) u=\lambda u+\mu u$

(8) $\lambda(u+v)=\lambda u+\lambda v$.

Definition 4.2. Let $C$ be an abstract cone and $d$ a metric on $C$. The pair $(C, d)$ will be called a metric cone if, for all $u, v, w \in C$ and $\lambda \in \mathbb{R}_{\geq 0}$,

$$
\begin{aligned}
d(0, \lambda u) & =\lambda d(0, u), \\
d(u+v, u+w) & \leq d(v, w) .
\end{aligned}
$$

We introduce the notation $\llbracket x \rrbracket:=d(0, x)$ for $x \in C$ and by $\mathbb{B}_{C}$ we will denote the open unit ball about $0 \in C$, i.e., $\mathbb{B}_{C}:=\{c \in C \mid \llbracket c \rrbracket<1\}$.

Similarly to Banach spaces, if a metric cone is complete, then absolutely convergent series always converge.

Lemma 4.3. Let $(C, d)$ be a complete metric cone. If a sequence $\left\{c_{i}\right\} \subseteq C$ is such that $\sum_{i=1}^{\infty} \llbracket c_{i} \rrbracket$ converges, then the series $\sum_{i=1}^{\infty} c_{i}$ converges in $C$.

Proof. Let $C$ be a complete metric cone. Let $\left\{c_{i}\right\} \subseteq C$ be such that $\sum_{i=1}^{\infty} \llbracket c_{i} \rrbracket<\infty$. From the definition of a metric cone, it is easily seen that $\left\{\sum_{i=1}^{n} c_{i}\right\}_{n \in \mathbb{N}}$ is a Cauchy sequence, and hence converges in $C$.

To establish our Open Mapping Theorem, we will employ the Baire Category Theorem in the form of Zabrelko's Lemma:

Lemma 4.4 (Zabreı̌ko's Lemma, [9, Lemma 1.6.3]). Every countably subadditive seminorm on a Banach space is continuous.

Finally, we will prove our main result of this section: 
Theorem 4.5 (Open Mapping Theorem for cone-valued correspondences). Let $C$ be a complete metric cone, $Y$ a Banach space and $D \subseteq Y$ a closed cone. Let $T: C \rightarrow Y$ be a continuous additive positively homogeneous map. If the correspondence $\Psi: C \rightarrow Y$ defined by $\Psi(c):=T c+D(c \in C)$ is surjective, (in the sense that, for every $y \in Y$, there exists some $c \in C$ such that $y \in \Psi(c))$, then $\Psi\left(\mathbb{B}_{C}\right) \subseteq Y$ is open.

Proof. Let $\Psi$, as defined, be surjective. Since $T$ is additive and $D$ is a cone, it is clear that $\Psi$ is an additive correspondence. We define the map $\rho: Y \rightarrow \mathbb{R}_{\geq 0}$ by

$$
\rho(y):=\inf \{\llbracket c \rrbracket \mid y \in \Psi(c)\}, \quad(y \in Y) .
$$

We note that $\rho$ is positively homogeneous, and, by additivity of $\Psi$, we see that $\rho$ is subadditive. Furthermore, the map $q: Y \rightarrow \mathbb{R}_{\geq 0}$, defined by $q(y):=\rho(y) \vee \rho(-y)$ for $y \in Y$, is a seminorm on $Y$.

We claim that that $q$ is countably subadditive.

Let $\left\{y_{n}\right\}_{n \in \mathbb{N}} \subseteq Y$ be such that the series $\sum_{n=1}^{\infty} y_{n}$ converges. If $\sum_{n=1}^{\infty} q\left(y_{n}\right)=\infty$ then $q\left(\sum_{n=1}^{\infty} y_{n}\right) \leq \sum_{n=1}^{\infty} q\left(y_{n}\right)$ holds trivially. We may therefore assume that $\sum_{n=1}^{\infty} q\left(y_{n}\right)<\infty$. Let $\kappa \in\{ \pm 1\}$ be such that $q\left(\sum_{n=1}^{\infty} y_{n}\right)=\rho\left(\kappa \sum_{n=1}^{\infty} y_{n}\right)$. Let $\varepsilon>0$ be arbitrary and, for each $n \in \mathbb{N}$, let $c_{n} \in C$ be such that $\kappa y_{n} \in \Psi\left(c_{n}\right)$ and $\llbracket c_{n} \rrbracket<\rho\left(\kappa y_{n}\right)+2^{-n} \varepsilon \leq q\left(y_{n}\right)+2^{-n} \varepsilon$. Then $\sum_{n=1}^{\infty} \llbracket c_{n} \rrbracket<\sum_{n=1}^{\infty} q\left(y_{n}\right)+\varepsilon$. Hence, by Lemma 4.3. the series $\sum_{n=1}^{\infty} c_{n}$ converges and $\llbracket \sum_{n=1}^{\infty} c_{n} \rrbracket<\sum_{n=1}^{\infty} q\left(y_{n}\right)+\varepsilon$.

For every $n \in \mathbb{N}$, we have $\kappa y_{n} \in \Psi\left(c_{n}\right)=T c_{n}+D$, i.e., there exists some $d_{n} \in D$ such that $\kappa y_{n}-T c_{n}=d_{n}$. Because $T$ is continuous and additive, the series $\sum_{n=1}^{\infty}\left(\kappa y_{n}-T c_{n}\right)$ converges to $\sum_{n=1}^{\infty} \kappa y_{n}-T\left(\sum_{n=1}^{\infty} c_{n}\right)$. But $\sum_{n=1}^{\infty} d_{n}=$ $\sum_{n=1}^{\infty} \kappa y_{n}-T\left(\sum_{n=1}^{\infty} c_{n}\right)$, and, in particular we note that the series $\sum_{n=1}^{\infty} d_{n}$ converges. Since $D$ is a closed cone, the series $\sum_{n=1}^{\infty} d_{n}$ converges to a point in $D$. Therefore

$$
\kappa \sum_{n=1}^{\infty} y_{n}=T\left(\sum_{n=1}^{\infty} c_{n}\right)+\sum_{n=1}^{\infty} d_{n} \in T\left(\sum_{n=1}^{\infty} c_{n}\right)+D,
$$

and

$$
q\left(\sum_{n=1}^{\infty} y_{n}\right)=\rho\left(\kappa \sum_{n=1}^{\infty} y_{n}\right) \leq \llbracket \sum_{n=1}^{\infty} c_{n} \rrbracket \leq \sum_{n=1}^{\infty} \llbracket c_{n} \rrbracket<\sum_{n=1}^{\infty} q\left(y_{n}\right)+\varepsilon .
$$

Since $\varepsilon>0$ was chosen arbitrarily, the claim that $q$ is countably subadditive follows. We conclude that $q$ is continuous by Zabrelko's Lemma (Lemma 4.4).

By subadditivity of $\rho$, for all $x, y \in Y$ we have $|\rho(x)-\rho(y)| \leq \max \{\rho( \pm(x-y))\}=$ $q(x-y)$, which implies that $\rho$ is also continuous, and finally, that $\rho^{-1}([0,1))=$ $\Psi\left(\mathbb{B}_{C}\right)$ is open.

\section{Strong Klee-Andô Theorems for coadditivity And Conormality}

We are now ready to establish our Strong Klee-Andô Theorems for conormality and coadditivity through an application of Theorem 4.5. Although our focus in this paper is on establishing a Klee-Andô Theorem for coadditivity (Corollary 5.5), for the sake of completeness and illustration we include a proof of a Klee-Andô Theorem conormality, Corollary 5.6 (also proven in [7]).

Theorem 5.1. Let $X$ be a Banach space and $\left\{C_{\omega}\right\}_{\omega \in \Omega}$ be an indexed collection of closed cones in $X$. 
(1) Let $Z$ be either of the spaces $\mathbf{c}(\Omega, X)$ or $\ell^{\infty}(\Omega, X)$, and let the correspondence $\Upsilon: Z \rightarrow X$ be defined by

$$
\Upsilon(\xi):=\bigcap_{\omega \in \Omega}\left(\xi_{\omega}+C_{\omega}\right) \quad(\xi \in Z) .
$$

If $\Upsilon$ is non-empty-valued, then there exists some $\alpha>0$ for which $\Upsilon$ is $\alpha$-bounded.

(2) Let the correspondence $\Delta: X \rightarrow \ell^{1}(\Omega, X)$ be defined by

$$
\Delta(x):=\left\{\xi \in \bigoplus_{\omega \in \Omega} C_{\omega} \subseteq \ell^{1}(\Omega, X) \mid \sum_{\omega \in \Omega} \xi_{\omega}=x\right\} \quad(x \in X) .
$$

If $\Delta$ is non-empty-valued, then there exists some $\alpha>0$ for which $\Delta$ is $\alpha$-bounded.

Proof. We prove (1) for the case that $Z=\ell^{\infty}(\Omega, X)$. The case where $Z=\mathbf{c}(\Omega, X)$ follows similarly.

Let $\left\{C_{\omega}\right\}_{\omega \in \Omega}$ be such that, for every $\xi \in \ell^{\infty}(\Omega, X)$, we have $\bigcap_{\omega \in \Omega}\left(\xi_{\omega}+C_{\omega}\right) \neq \emptyset$. Define $D:=\bigoplus_{\omega \in \Omega}\left(-C_{\omega}\right) \subseteq \ell^{\infty}(\Omega, X)$ and $T: X \rightarrow \ell^{\infty}(\Omega, X)$ as $T x:=(\omega \mapsto x)$ for all $x \in X$ and $\omega \in \Omega$. The cone $D$ is closed in $\ell^{\infty}(\Omega, X)$, and, since $T$ is a linear isometry, it is clear that $T$ is continuous, additive and positively homogeneous. We define $\Psi: X \rightarrow \ell^{\infty}(\Omega, X)$ by $\Psi(x):=T x+D$ for all $x \in X$. It is easily seen that $\Psi$ is surjective: Let $\xi \in \ell^{\infty}(\Omega, X)$ be arbitrary and choose $x \in \bigcap_{\omega \in \Omega}\left(\xi_{\omega}+C_{\omega}\right) \neq \emptyset$, then $\xi \in T x+D$. Now, by our Open Mapping Theorem (Theorem 4.5), $\Psi\left(\mathbb{B}_{X}\right)$ is open in $\ell^{\infty}(\Omega, X)$. Let $\beta>0$ be such that $\beta \mathbb{B}_{\ell^{\infty}(\Omega, X)} \subseteq \Psi\left(\mathbb{B}_{X}\right)$. Then, for every $\xi \in \ell^{\infty}(\Omega, X)$ and $\varepsilon>0$, there exists some $w \in \mathbb{B}_{X}$ such that $\beta \xi /(1+\varepsilon \beta)\|\xi\|_{\infty} \in$ $\Psi(w)=T w+D$. Setting $x:=\left(\beta^{-1}+\varepsilon\right)\|\xi\|_{\infty} w$ we obtain $\xi \in \Psi(x)$, implying $x \in \bigcap_{\omega \in \Omega}\left(\xi_{\omega}+C_{\omega}\right)$, and $\|x\| \leq\left(\beta^{-1}+\varepsilon\right)\|\xi\|_{\infty}$. I.e., setting $\alpha:=\beta^{-1}$, we have, for any $\xi \in \ell^{\infty}(\Omega, X)$ and $\varepsilon>0$, that $\Upsilon(\xi) \cap(\alpha+\varepsilon)\|\xi\|_{\infty} \mathbf{B}_{X} \neq \emptyset$. We conclude that the correspondence $\Upsilon$ is $\alpha$-bounded.

We prove (2). Let $\left\{C_{\omega}\right\}_{\omega \in \Omega}$ be such that for every $x \in X$, there exists some $\xi \in \bigoplus_{\omega \in \Omega} C_{\omega} \subseteq \ell^{1}(\Omega, X)$ such that $x=\sum_{\omega \in \Omega} \xi_{\omega}$. We define $C:=\bigoplus_{\omega \in \Omega} C_{\omega} \subseteq$ $\ell^{1}(\Omega, X)$ and $\Sigma: C \rightarrow X$ by $\Sigma \xi:=\sum_{\omega \in \Omega} \xi_{\omega}$ for $\xi \in \ell^{1}(\Omega, X)$. Let $\Psi: \ell^{1}(\Omega, X) \rightarrow X$ be defined by $\Psi(\xi):=\Sigma \xi+\{0\}$. The cone $C$ is a complete metric cone with the metric induced by the $\ell^{1}$-norm, and furthermore, the map $\Sigma$ is surjective, continuous, additive and positively homogeneous. Therefore, by our Open Mapping Theorem (Theorem 4.5$), \Psi\left(\mathbb{B}_{C}\right)$ is an open set. Let $\beta>0$ be such that $\beta \mathbb{B}_{X} \subseteq \Psi\left(\mathbb{B}_{C}\right)$. Then, for every $x \in X$ and $\varepsilon>0$, there exists some $\eta \in \mathbb{B}_{C}$ such that $\beta x /(1+\varepsilon \beta)\|x\| \in \Psi(\eta)=\sum_{\omega \in \Omega} \eta_{\omega}+\{0\}$. Setting $\xi:=\left(\beta^{-1}+\varepsilon\right)\|x\| \eta$, we obtain $x \in \Psi(\xi)$, implying $x=\sum_{\omega \in \Omega} \xi_{\omega}$, and $\|\xi\|_{1} \leq\left(\beta^{-1}+\varepsilon\right)\|x\|$. I.e., setting $\alpha:=\beta^{-1}$, for any $x \in X$ and $\varepsilon>0$, we obtain $\Delta(x) \cap(\alpha+\varepsilon)\|x\| \mathbf{B}_{\ell^{1}(\Omega, X)} \neq \emptyset$. We conclude that the correspondence $\Delta$ is $\alpha$-bounded.

We now apply Proposition 3.3 to show that certain correspondences related to $\Upsilon$ and $\Delta$ are lower hemicontinuous.

Corollary 5.2. Let $X$ be a Banach space and $\left\{C_{\omega}\right\}_{\omega \in \Omega}$ an indexed collection of closed cones.

(1) Let $Z$ be either of the spaces $\mathbf{c}(\Omega, X)$ or $\ell^{\infty}(\Omega, X)$. If $\Upsilon: Z \rightarrow X$, as defined in Theorem 5.1, is non-empty-valued, then there exists a constant 
$\alpha>0$ such that, for every $\varepsilon>0$, the correspondence $\Upsilon_{\varepsilon}: \mathbf{S}_{Z} \rightarrow X$, defined by

$$
\Upsilon_{\varepsilon}(\xi):=\Upsilon(\xi) \cap(\alpha+\varepsilon) \mathbf{B}_{X} \quad\left(\xi \in \mathbf{S}_{Z}\right),
$$

is non-empty-closed-and convex-valued, and is lower hemicontinuous.

(2) If $\Delta: X \rightarrow \ell^{1}(\Omega, X)$, as defined in Theorem [5.1] is non-empty-valued, then there exists a constant $\alpha>0$ such that, for every $\varepsilon>0$, the correspondence $\Delta_{\varepsilon}: \mathbf{S}_{X} \rightarrow \ell^{1}(\Omega, X)$, defined by

$$
\Delta_{\varepsilon}(x):=\Delta(x) \cap(\alpha+\varepsilon) \mathbf{B}_{\ell^{1}(\Omega, X)} \quad\left(x \in \mathbf{S}_{X}\right),
$$

is non-empty-closed-and convex-valued, and is lower hemicontinuous.

Proof. We prove (1). By Theorem 5.1(1), there exists some $\alpha>0$ for which $\Upsilon$ is $\alpha$-bounded. It is then clear that $\Upsilon_{\varepsilon}$ is then non-empty-, closed-and convex-valued for every $\varepsilon>0$. It is easily seen that $\Upsilon$ is additive, so that, by Proposition $3.3 . \Upsilon_{\varepsilon}$ is lower hemicontinuous for every $\varepsilon>0$.

We prove (2). By Theorem [5.1 (2), there exists some $\alpha>0$ for which $\Delta$ is $\alpha$ bounded. Again, it is clear that $\Delta_{\varepsilon}$ is non-empty-, closed-and convex-valued for every $\varepsilon>0$. That $\Delta$ is additive is easily seen, so that, by Proposition 3.3 . $\Delta_{\varepsilon}$ is lower hemicontinuous for every $\varepsilon>0$.

We will now apply Michael's Selection Theorem to obtain continuous selections of $\Delta$ and $\Upsilon$.

Theorem 5.3 (Michael's Selection Theorem [2, Theorem 17.66]). A lower hemicontinuous correspondence from a paracompact space into a Banach space with nonempty, closed and convex values admits a continuous selection.

Corollary 5.4. Let $X$ be a Banach space and $\left\{C_{\omega}\right\}_{\omega \in \Omega}$ an indexed collection of closed cones.

(1) Let $Z$ be either of the spaces $\mathbf{c}(\Omega, X)$ or $\ell^{\infty}(\Omega, X)$. If $\Upsilon: Z \rightarrow X$, as defined in Theorem 5.1, is non-empty-valued, then there exists a constant $\alpha>0$ such that, for every $\varepsilon>0$, there exists a continuous positively homogeneous selection $v: Z \rightarrow X$ of $\Upsilon$ such that $\|v(\xi)\| \leq(\alpha+\varepsilon)\|\xi\|_{\infty}$ for every $\xi \in Z$.

(2) If $\Delta: X \rightarrow \ell^{1}(\Omega, X)$, as defined in Theorem 5.1, is non-empty-valued, then there exists a constant $\alpha>0$ such that, for every $\varepsilon>0$, there exists a continuous positively homogeneous selection $\delta: X \rightarrow \ell^{1}(\Omega, X)$ of $\Delta$ such that $\|\delta(x)\|_{1} \leq(\alpha+\varepsilon)\|x\|$ for every $x \in X$.

Proof. Before we begin, we note that all metric spaces are paracompact [14], so that, for any normed space $N$, its unit sphere $\mathbf{S}_{N}$ is paracompact with the metric induced from the norm.

We prove (1). By Corollary 5.2(1) and Michael's Selection Theorem (Theorem [5.3), for every $\varepsilon>0$, there exists a continuous selection $\underline{v}: \mathbf{S}_{Z} \rightarrow X$ of $\Upsilon_{\varepsilon}: \mathbf{S}_{Z} \rightarrow X$. We define $v$ by

$$
v(\xi):=\left\{\begin{array}{ll}
\|\xi\|_{\infty} \underline{v}\left(\frac{\xi}{\|\xi\|_{\infty}}\right) & \xi \neq 0 \\
0 & \xi=0
\end{array} \quad(\xi \in Z),\right.
$$

which is clearly positively homogeneous. That $v$ is continuous follows from an application of the reverse triangle inequality while keeping the boundedness of $v$ in mind. 
We prove (2). By Corollary [5.2(2) and Michael's Selection Theorem (Theorem [5.3), for every $\varepsilon>0$, there exists a continuous selection $\underline{\delta}: \mathbf{S}_{X} \rightarrow \ell^{1}(\Omega, X)$ of $\Delta_{\varepsilon}: \mathbf{S}_{X} \rightarrow \ell^{1}(\Omega, X)$. By defining

$$
\delta(x):=\left\{\begin{array}{ll}
\|x\| \underline{\delta}\left(\frac{x}{\|x\|}\right) & x \neq 0 \\
0 & x=0
\end{array} \quad(x \in X),\right.
$$

we are done, noting that positive homogeneity of $\delta$ is clear, and that continuity follows again through application of the reverse triangle inequality and the boundedness of $\delta$.

The following two results, our Strong Klee-Andô Theorems, now follow from Corollary 5.4 It is trivial that $(3) \Rightarrow(2) \Rightarrow(1)$ in both results below. The implication $(1) \Rightarrow(3)$ in both results below easily follow by fixing some $\varepsilon>0$, say $\varepsilon:=1$, and applying Corollary 5.4 .

Corollary 5.5 (Strong Klee-Andô Theorem for coadditivity). Let $X$ be a Banach space and $\left\{C_{\omega}\right\}_{\omega \in \Omega}$ an indexed collection of closed cones in $X$. Let $Z$ be either of the spaces $\mathbf{c}(\Omega, X)$ or $\ell^{\infty}(\Omega, X)$. Then the following are equivalent:

(1) For every $\xi \in Z$, the intersection $\bigcap_{\omega \in \Omega}\left(\xi_{\omega}+C_{\omega}\right)$ is non-empty.

(2) There exists an $\alpha>0$ such that, for every $\xi \in Z$, there exists some $y \in$ $\bigcap_{\omega \in \Omega}\left(\xi_{\omega}+C_{\omega}\right)$ with $\|y\| \leq \alpha\|\xi\|_{\infty}$.

(3) There exists an $\alpha>0$ and a continuous positively homogeneous map $v$ : $Z \rightarrow X$ such that, for every $\xi \in Z$, we have $v(\xi) \in \bigcap_{\omega \in \Omega}\left(\xi_{\omega}+C_{\omega}\right)$ and $\|v(\xi)\| \leq \alpha\|\xi\|_{\infty}$.

Corollary 5.6 (Strong Klee-Andô Theorem for conormality). Let $X$ be a Banach space and $\left\{C_{\omega}\right\}_{\omega \in \Omega}$ an indexed collection of closed cones in $X$. The following are equivalent:

(1) For every $x \in X$, there exists a decomposition $x=\sum_{\omega \in \Omega} c_{\omega}$, with $c_{\omega} \in C_{\omega}$ for every $\omega \in \Omega$, and satisfying $\sum_{\omega \in \Omega}\left\|c_{\omega}\right\|<\infty$.

(2) There exists an $\alpha>0$ such that, for every $x \in X$, there exists a decomposition $x=\sum_{\omega \in \Omega} c_{\omega}$, with $c_{\omega} \in C_{\omega}$ for every $\omega \in \Omega$, and satisfying $\sum_{\omega \in \Omega}\left\|c_{\omega}\right\| \leq \alpha\|x\|$.

(3) There exists an $\alpha>0$ and, for every $\omega \in \Omega$, there exists a continuous positively homogeneous map $\delta_{\omega}: X \rightarrow C_{\omega}$ such that, for every $x \in X$, we have $x=\sum_{\omega \in \Omega} \delta_{\omega}(x)$ and $\sum_{\omega \in \Omega}\left\|\delta_{\omega}(x)\right\| \leq \alpha\|x\|$.

\section{REFERENCES}

1. I. Aharoni and J. Lindenstrauss, Uniform equivalence between Banach spaces, Bull. Amer. Math. Soc. 84 (1978), no. 2, 281-283.

2. C.D. Aliprantis and K.C. Border, Infinite dimensional analysis, third ed., Springer, Berlin, 2006.

3. C.D. Aliprantis and R. Tourky, Cones and duality, American Mathematical Society, Providence, RI, 2007.

4. T. Andô, On fundamental properties of a Banach space with a cone, Pacific J. Math. 12 (1962), 1163-1169.

5. Y. Benyamini and J. Lindenstrauss, Geometric nonlinear functional analysis. Vol. 1, American Mathematical Society, Providence, RI, 2000.

6. M. de Jeu and M. Messerschmidt, Crossed products of Banach algebras. III, arXiv:1306.6290. 
7. _ A strong open mapping theorem for surjections from cones onto Banach spaces, Adv. Math. 259 (2014), 43-66.

8. V.L. Klee, Boundedness and continuity of linear functionals, Duke Math. J. 22 (1955), 263269.

9. R.E. Megginson, An introduction to Banach space theory, Springer-Verlag, New York, 1998.

10. M. Messerschmidt, Geometric duality theory of cones in dual pairs of vector spaces, J. Funct. Anal. 269 (2015), no. 7, 2018-2044.

11. N Normality of spaces of operators and quasi-lattices, Positivity 19 (2015), no. 4, 695724.

12. _ A pointwise Lipschitz selection theorem, Set-Valued Var. Anal. (2017), To appear in print. Online: https://doi.org/10.1007/s11228-017-0455-2.

13. H.H. Schaefer, Banach lattices and positive operators, Springer-Verlag, New York-Heidelberg, 1974.

14. A.H. Stone, Paracompactness and product spaces, Bull. Amer. Math. Soc. 54 (1948), 977-982.

Miek Messerschmidt; Department of Mathematics and Applied Mathematics; UniverSity of Pretoria; Private bag X20 Hatfield; 0028 Pretoria; South Africa

E-mail address: mmesserschmidt@gmail.com 